\title{
Las nuevas fachadas autoportantes de ladrillo cara vista
}

\section{The new self-supporting face brick façades}

\author{
C. del Río ${ }^{(*)}$
}

\section{RESUMEN}

La 'Fachada Autoportante' de ladrillo cara vista es el resultado de una profunda reflexión sobre las diferentes soluciones de fachada que se han utilizado a lo largo del siglo pasado para los edificios con estructura porticada, y se fundamenta en el aprovechamiento del potencial que tienen los materiales cerámicos cuando trabajan a compresión. La solución constructiva consiste en disponer la hoja exterior del cerramiento pasante por delante de la estructura del edificio en la totalidad de su espesor, utilizando el propio muro de ladrillo como estructura portante de sí mismo. Este tipo constructivo elimina los puentes térmicos en el encuentro con los forjados, y es más eficaz para el comportamiento mecánico que las soluciones convencionales, puesto que el efecto beneficioso del propio peso contribuye a la estabilidad frente a las acciones horizontales.

Palabras clave: ladrillo cara vista; autoportante; aislamiento térmico; eficiencia energética; fachada ventilada.

\section{ABSTRACT}

The 'Self-Supporting' face brick façade is the result of a profound reflection on the different façade solutions that have been used throughout the last century for the pillar-structured buildings, and is based on the utilization of the potential that ceramic materials have when they work in compression. The construction solution consists in placing the entire outer sheet of the enclosure ahead of the building's structure, so as to use the brick wall itself as its own supporting structure. This construction type eliminates the thermal bridges at façade-slab joints, and is more effective regarding mechanical behaviour than conventional solutions, since the own-weight effect contributes beneficially to stability against horizontal actions.

Keywords: face brick; shelf-supporting; thermal insulation; energy efficiency; ventilated façade.

(*) Universidad Politécnica de Madrid. Madrid (España).

Persona de contacto/Corresponding author: mcdelrio@telefonica.net (C. del Río)

Cómo citar este artículo/Citation: Del Río, C. (2016). Las nuevas fachadas autoportantes de ladrillo cara vista. Informes de la Construcción, 68(544): e171, doi: http://dx.doi.org/10.3989/ic.15.165.m15.

Copyright: (C) 2016 CSIC. Licencia / License: Salvo indicación contraria, todos los contenidos de la edición electrónica de Informes de la Construcción se distribuyen bajo una licencia de uso y distribución Creative Commons Attribution License (CC BY) Spain 3.o. 


\section{INTRODUCCIÓN}

En la actualidad, el uso de muros de fábrica de ladrillo como solución a la estructura global del edificio es marginal, restringido a construcciones modestas de una o dos plantas. Pero en cambio, los elementos secundarios, como tabiques, petos y fachadas, es habitual resolverlos con muros de fábrica.

Las fachadas de ladrillo cerámico representan una de las unidades constructivas más importantes en las obras de edificación de nuestro país. Pero, sin embargo, sus prestaciones, soluciones y especificaciones técnicas no han sido suficientemente consideradas en la normativa hasta la aprobación del Código Técnico de la Edificación (en adelante, CTE).

En la construcción tradicional se procedía con reglas de buena práctica, casi siempre de índole geométrica. Pero el cambio de sistemas estructurales de muros de carga por sistemas de pórticos ha hecho necesario generar soluciones para los que no había reglas. La forma de concebir, proyectar y construir los cerramientos de fachada evolucionó durante la segunda mitad del siglo pasado sin el respaldo que supone una evolución en el mismo sentido de la normativa o, al menos, de sus reglas de aplicación'․

La elaboración del CTE supuso un considerable esfuerzo por cubrir lagunas y dar respaldo legal a determinados materiales, sistemas y tipos constructivos que habían permanecido durante décadas en un vacío legal, entre los cuales estaban las fachadas de los edificios de pisos.

En este marco de cambio normativo en el ámbito de la edificación en España se gestó el tipo constructivo de 'Fachada Autoportante' impulsado por la Sección Cara Vista de Hispalyt, para afrontar el reto de proponer soluciones competitivas y de elevadas prestaciones para las fachadas de ladrillo tradicional.

La 'Fachada Autoportante' debe su nombre a la posición del muro que constituye la hoja exterior del cerramiento de fachada, que se construye separado de la estructura del edificio, gravitando sobre sí mismo en toda la altura que permite el cálculo estructural. Este tipo constructivo es el resultado de una rigurosa y sistemática exploración de las diferentes soluciones de fachada que surgieron en el siglo pasado (1), analizadas bajo la óptica de las exigencias de naturaleza mecánica para cumplir el requisito esencial de Resistencia y Estabilidad. Se fundamenta en el aprovechamiento del potencial estructural que tienen los materiales cerámicos cuando trabajan a compresión, lo que permite asignar al muro de ladrillo un papel esencial en el comportamiento mecánico de la fachada.

El sistema convencional que se ha venido usando para construir las fachadas de ladrillo, cuya estabilidad se confía a su confinamiento en los forjados, así como los sistemas que utilizan dispositivos específicos de apoyo (tales como angulares, ménsulas o consolas) para el sostén de la fachada en cada planta del edificio, tienen en común la concepción del muro de ladrillo como un elemento inerte, es decir, como un peso muerto que es necesario sostener y trasmitir a la estructura portante del edificio a intervalos relativamente pequeños. Por el contrario, la 'Fachada Autoportante' parte de la concepción del propio muro de ladrillo como un elemento activo y esencial en el comportamiento mecánico, utilizando su capacidad resistente a compresión para soportarse a sí mismo; lo que permite, además, contabilizar la contribución del propio peso en la resistencia y estabilidad ante las acciones horizontales.

Recientemente, las normas de edificación han experimentado otro cambio importante en el ámbito relacionado con la eficiencia energética de los edificios, y han sido adaptadas a los criterios de la Unión Europea (2) para el cumplimiento, entre otros, del Objetivo 2O/2O/2O $\mathrm{O}^{2}$. La actualización de 2013 del Documento Básico "Ahorro de Energía» (en adelante, $D B H E$ ) del CTE constituye sólo la primera etapa de aproximación a este ambicioso objetivo, y aun así supone un considerable incremento de las prestaciones exigidas a los edificios en materia de la demanda del consumo energético, y una modificación del enfoque a utilizar para su análisis. Esta circunstancia afecta directamente a la unidad constructiva de los cerramientos de fachada, como parte integrante de la envolvente térmica de los edificios.

En este sentido, la 'Fachada Autoportante', que surgió para dar respuesta a las deficiencias de índole mecánica que tenían las soluciones convencionales, se está imponiendo también para dar respuesta al incremento de las prestaciones higrotérmicas exigidas a los edificios, puesto que habilita la posibilidad de dar continuidad al aislamiento de la fachada, evitando los puentes térmicos.

\section{COMPORTAMIENTO MECÁNICO DE LAS FACHADAS DE LADRILLO CERÁMICO}

Si se exploran los diferentes sistemas que se han utilizado históricamente para resolver los cerramientos de fachada de los edificios, se pueden agrupar en dos tipos fundamentales.

Un primer tipo corresponde a las fachadas concebidas como muros de carga que se sustentan sobre sí mismas en toda su altura. Tradicionalmente se configuraban como sistemas monocapa, con muros de gran espesor, en los que el propio muro resuelve todas las prestaciones exigidas al cerramiento, tanto las de naturaleza mecánica, debidas al transporte de las cargas de forjados, como las de naturaleza funcional, relacionadas con los requisitos de aislamiento.

El segundo tipo corresponde a las fachadas concebidas como una piel exterior del edificio, que habitualmente se sustentan en los forjados de cada planta; bien por confinamiento ${ }^{3}$, cuando el material tolera aceptablemente los esfuerzos de compresión; o bien por suspensión, como es el caso de los muros cortina, cuando el material resiste esfuerzos de tracción. Estos sistemas son multicapa, con varias hojas y barreras

\footnotetext{
${ }^{1}$ Aunque el CTE, publicado en 2006, sí cubre, plantea y hasta resuelve el análisis de todo tipo de muros de fábrica, todavía subsisten, por inercia, prácticas y criterios «para no calcular» las fachadas, algunos de ellos infundados o poco acertados, cuando no totalmente equívocos. 2 El Objetivo 20/20/20 supone el compromiso, para el año 2020 de reducir en un 20 \% las emisiones de CO2 respecto al año 1990 , de reducir en un 20 \% el consumo de energía primaria también respecto al año 1990 y que el 20 \% de la energía consumida proceda de energías renovables.

3 La sustentación de un muro por confinamiento se consigue retacando la última hilada contra la cara inferior del forjado.
} 
específicas para cada una de las prestaciones exigidas al cerramiento. En este grupo se pueden incluir la mayoría de los cerramientos de los edificios modernos con estructura porticada.

Cuando las fachadas de los edificios dejaron de ser muros de carga y pasaron a ser concebidas como una piel exterior, su comportamiento mecánico cambió sustancialmente. Dejaron de tener la acción gravitatoria procedente de los forjados, esencial para su estabilidad y, sin embargo, debían seguir dando respuesta mecánica a las acciones horizontales, fundamentalmente a la acción de viento. El objetivo del análisis estructural de estos muros ya no es mantener el valor y trayectoria de la compresión dentro de un rango aceptable, sino que pasó a ser la consecución de una adecuada respuesta ante las acciones horizontales, cuyo efecto se volvió mucho más agresivo por el hecho de no disponer de una buena parte de la carga gravitatoria estabilizante que antes tenían.

\subsection{Descripción de la fachada confinada de ladrillo (sistema convencional)}

El sistema constructivo que propició resolver esta nueva circunstancia en las fachadas de los edificios con estructura de pórticos, sin necesidad de recurrir a la escasa y poco fiable resistencia a tracción del material cerámico, es el que se ha llamado aquí sistema convencional (Figura 1), que consiste en confinar los muros de fachada entre los forjados de piso cargados, asegurando el contacto físico entre ambos elementos, para conseguir las reacciones de empuje en los extremos del muro que garanticen su estabilidad ante la acción horizontal de viento.

Aunque el traslado de la acción horizontal a los forjados en los que se sustenta la fachada supone una solicitación de flexión; sin embargo, debido a que el muro es un elemento extenso cuyas secciones extremas están confinadas, el traba- jo estructural se puede conseguir prescindiendo de las tracciones. Para ello es suficiente que sea posible encajar, en el espesor del muro, un arco que reproduzca el antifunicular de las carga, siempre que los extremos estén suficientemente coaccionados para poder generar la reacción oblicua correspondiente.

Este funcionamiento para el traslado de acciones horizontales, con solicitación exclusivamente de compresión, es el conocido como «efecto arco», y se desarrolla espontáneamente siempre que las condiciones de sustentación permitan generar empujes, puesto que se trata del funcionamiento estructural más rentable energéticamente, para valores significativos de acción horizontal, en materiales que tienen una escasa resistencia a tracción (3).

\subsubsection{Modelos para el análisis de un muro confinado}

Los modelos disponibles para el análisis de una fachada confinada de ladrillo ante la acción de viento son los que corresponden al traslado de una fuerza horizontal a los extremos del muro 4 (Figura 2).

En el modelo viga las tensiones proceden de considerar la pieza como doblemente sustentada ${ }^{5}$ en los extremos, con tracciones y compresiones de valor igual en cada sección y máximos a media altura. La reacción en los extremos, $H$, es sólo de cortante y se resiste por la capacidad al corte de la junta del muro contra el forjado. Las trayectorias de cada solicitación son, para carga horizontal uniforme, dos parábolas simétricas que, a media altura, para tensión lineal, operan con un brazo mecánico, $z$, de valor igual a dos tercios del espesor del muro.

Ante la presencia de una compresión en los extremos, por ejemplo, debido a la existencia de una carga en el forjado, la reacción $R$ puede ser oblicua, y las trayectorias de tracción y

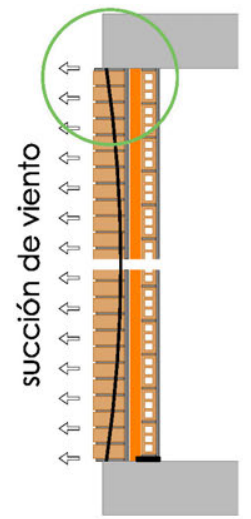

ESQUEMA EN SECCIÓN

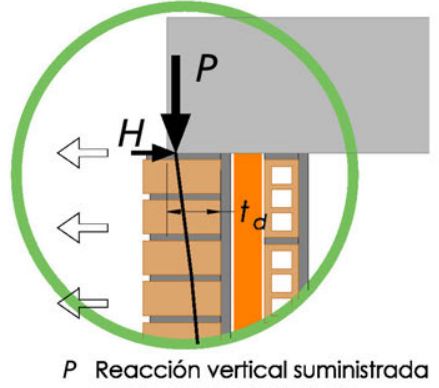
por la carga del forjado

$H$ Reacción horizontal suministrada por la junta de mortero

$t_{d}$ Espesor de cálculo a succión

COMPORTAMIENTO MECÁNICO

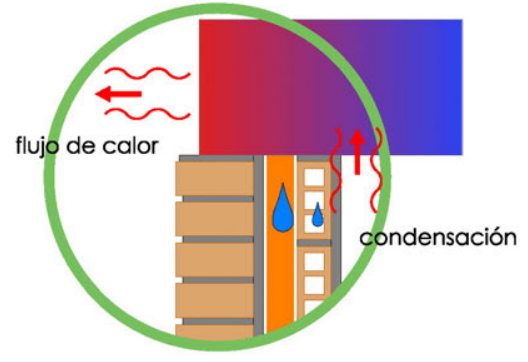

Puente térmico en el canto del forjado Riesgo de humedades de condensación COMPORTAMIENTO TÉRMICO

Figura 1. Fachada de ladrillo confinada entre forjados (sistema convencional).

\footnotetext{
4 La acción gravitatoria en un muro confinado es sólo la procedente del propio peso del paño entre forjados, y no tiene entidad suficiente para producir tensiones elevadas. Por otra parte, la carga vertical, cuando produce tensiones pequeñas en relación con la resistencia del material, al combinarse con la acción horizontal, tiene efecto favorable, razón por la cual se puede despreciar en el análisis. La formulación que presenta el Documento Básico «Seguridad estructural: Fábrica» del CTE en el artículo 5·4.4, expresión (5·32) procede de despreciar el propio peso del muro.

5 Utilizamos el término «sustentada» en lugar de «apoyada» para no inducir a error, puesto que el análisis que se plantea es frente a una acción horizontal y, por consiguiente, las reacciones en la sustentación también son horizontales. El término «apoyo» tiene una connotación de sustentación ante carga gravitatoria y podría resultar equívoco.
} 


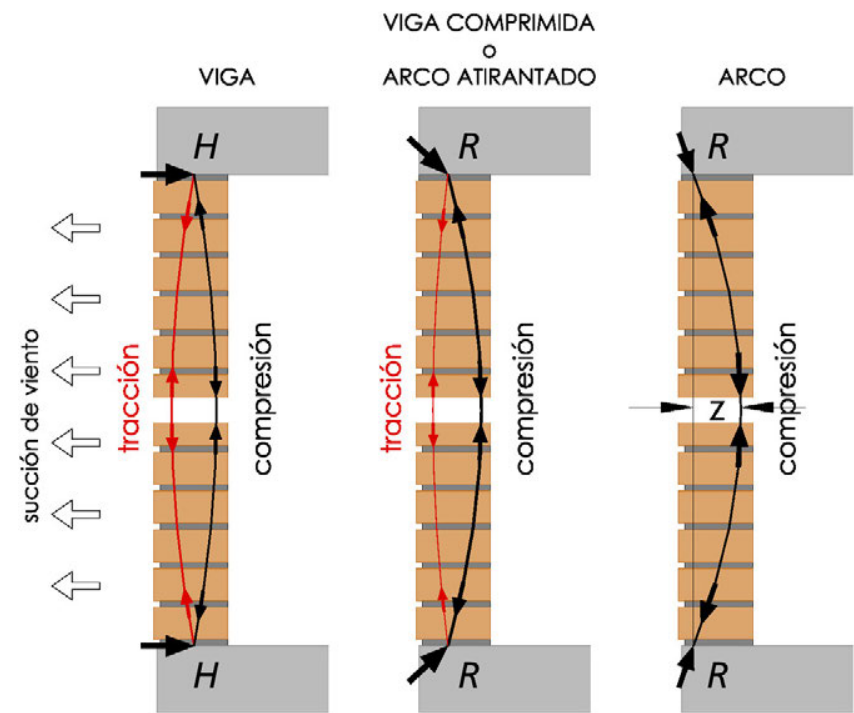

Figura 2. Modelos para el análisis de una fachada confinada ante acción horizontal.

compresión pasan a ser asimétricas, con tracción reducida y compresión aumentada respecto del caso anterior. El modelo corresponde al de una viga comprimida o un arco atirantado, con las resultantes de tensiones en los extremos desplazadas a sotavento.

Si la carga gravitatoria de los forjados tiene un valor suficiente, puede anular las tracciones por completo, quedando sólo la trayectoria parabólica de la compresión (que pasa a ser la trayectoria de carga). Esta situación corresponde al modelo arco ${ }^{6}$.

Si para una acción horizontal dada compiten varios modelos alternativos, como viga contra arco, o arcos con mayor tensión pero menor amplitud que otros, el modelo de respuesta que realmente se genera es el que produce el mínimo trabajo de deformación.

El modelo viga, en general, es más rígido que el modelo arco, pero fracasa en cuanto se alcanzan tensiones de tracción insoportables para el material. En edificios de piso, con alturas de planta del orden de $3 \mathrm{~m}$, esto sucede para una acción de viento del orden de la cuarta parte de los valores habituales (3).

Por consiguiente, el modelo que da cuenta del equilibrio ante la acción de viento en los muros de fachada confinados, eludiendo las tracciones, es el modelo arco, con un brazo mecánico, $z$, igual a dos tercios del espesor del muro, obtenido por un simple cálculo de mínimos (3).

La condición de resistencia se deduce de comparar la máxima solicitación producida por la acción de viento con la capacidad resistente del muro, analizado como arco:

Momento flector máximo por viento:

$$
M_{S d}=\frac{w_{d} \cdot h^{2}}{8}
$$

Capacidad resistente del arco:

$$
M_{R d}=U \cdot z=f_{d} \cdot \frac{1}{3} t_{d} \cdot \frac{2}{3} t_{d}=\frac{2}{9} \cdot f_{d} \cdot t_{d}^{2}
$$

Condición de resistencia:

$$
M_{S d} \leq M_{R d} ; w_{d} \leq \frac{16}{9} \cdot f_{d} \cdot \frac{t_{d}^{2}}{h^{2}}
$$

siendo:

$w_{d} \quad$ valor de cálculo de la acción horizontal;

$f_{d} \quad$ valor de cálculo de la resistencia a compresión del muro;

$t_{d} \quad$ espesor de cálculo del muro 7

$h \quad$ altura libre del muro entre forjados;

$U$ resultante de la fuerza de compresión $\left(=f_{d} \cdot t_{d} / 3\right)$;

$z \quad$ brazo mecánico del $\operatorname{arco}\left(=2 / 3 \cdot t_{d}\right)$.

La condición de resistencia [3] se puede expresar en términos de la esbeltez del muro:

$$
\lambda^{2} \leq \frac{16}{9} \cdot \frac{f_{d}}{w_{d}}
$$

siendo:

$\lambda \quad$ esbeltez del muro $\left(=h / t_{d}\right)$.

En la expresión [4] se puede ver que la esbeltez del muro interviene elevada al cuadrado, lo que pone de manifiesto que, incluso en un análisis en primer orden, las fachadas confinadas de ladrillo son extraordinariamente sensibles a la condición de entrega en los forjados ${ }^{8}$. Esta restricción, relativa a la esbeltez, es todavía mayor si se tienen en cuenta los efectos de segundo orden, que no han sido considerados en el análisis anterior.

\section{Modelo arco. Análisis en segundo orden}

Los efectos de segundo orden tienen como consecuencia que el fenómeno no sea lineal, como pudiera parecer observando la condición de resistencia [4]; de manera que, si se modifica

\footnotetext{
${ }^{6}$ La serie de modelos de la Figura 2 se puede ampliar, con situaciones en las que existen cargas en los extremos de valor superior al estricto que se precisa para neutralizar las tracciones. Estas situaciones corresponderían al modelo de arco cargado, con brazo mecánico, $z$, menor, y trayectoria más tendida. En el límite el arco deviene en una recta.

7 En el cómputo del espesor del muro se debe tomar como espesor de cálculo, td, el ancho del sólido capaz en el que se puede inscribir el arco, es decir, el espesor total para presión, y el ancho de la entrega en el forjado para succión.

${ }^{8}$ Nótese que en un muro con entrega total en los forjados, la acción que condiciona la resistencia es la presión de viento, puesto que habitualmente tiene valores superiores a la succión. Sin embargo, con entrega parcial, el efecto determinante es la succión.
} 
la esbeltez, no se pueden deducir proporcionalmente los resultados.

Considerar los efectos de segundo orden supone analizar el elemento con la geometría que le corresponde tras la deformación. La acción horizontal, actuando sobre el muro, lo deforma; de manera que el brazo mecánico, $z$, considerado inicialmente se reduce, disminuyendo en la misma proporción la capacidad resistente. Esto supone un proceso iterativo que puede no llegar a converger ${ }^{9}$.

El procedimiento de análisis en segundo orden consiste en corregir el espesor del muro, deduciendo del canto total el valor de la flecha inicial $\delta_{0}$. Con el espesor corregido se realiza un nuevo análisis, obteniéndose un nuevo valor de flecha $\delta_{1}$, que implica una nueva corrección del espesor, y así sucesivamente hasta llegar a la situación límite (véase la Figura 3). El proceso es el siguiente:

Deformación del arco:

$$
\delta=\frac{\varepsilon}{4 \cdot z} \cdot h^{2}
$$

siendo:

e deformación unitaria $(=\sigma / E)$;

$\sigma \quad$ tensión de compresión $(=U / b)$;

$U$ resultante de la fuerza de compresión ( $=M_{S d} / z$; con $M_{S d}$ $\left.=w_{d} \cdot h^{2} / 8\right)$;

$w_{d} \quad$ valor de cálculo de la acción de viento;

$h \quad$ altura libre del muro entre forjados;

$b \quad$ ancho de la biela comprimida $\left(=t_{d} / 3\right)$;

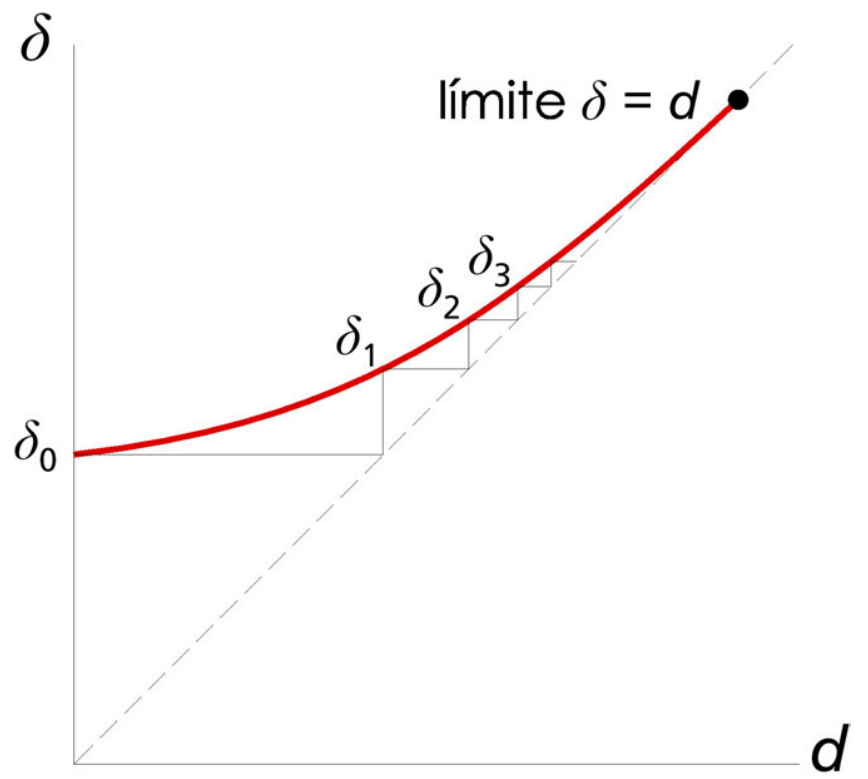

Figura 3. Deformación del arco en segundo orden. $z \quad$ brazo mecánico del $\operatorname{arco}\left(=2 / 3 \cdot t_{d}\right)$

$t_{d} \quad$ espesor de cálculo del muro;

E módulo de elasticidad de la fábrica.

Sustituyendo los valores de $\varepsilon$ y $z$ en [5], se obtiene la flecha del $\operatorname{arco}, \delta_{0}$, en el momento inicial, con el muro sin deformar:

$$
\delta_{\mathrm{o}}=\frac{w_{d} \cdot h^{4}}{32 \cdot\left(\frac{2}{3}\right)^{2} \cdot\left(\frac{1}{3}\right) \cdot t_{d}^{3} \cdot E}
$$

Posteriormente se calcularía un segundo valor $\delta_{1}$, con el espesor disminuido en $\delta_{0}$ :

$$
\delta_{1}=\frac{w_{d} \cdot h^{4}}{32 \cdot\left(\frac{2}{3}\right)^{2} \cdot\left(\frac{1}{3}\right) \cdot\left(t_{d}-\delta_{\mathrm{o}}\right)^{3} \cdot E}
$$

$\mathrm{Y}$ así sucesivamente. El proceso termina cuando la flecha, $\delta$, coincide con la deformación tota, $d$, lo que permite deducir la función $\delta-d$ :

$$
\delta=\frac{27 \cdot w_{d} \cdot h^{4}}{128 \cdot\left(t_{d}-d\right)^{3} \cdot E}
$$

En el límite, $\delta$ es igual a $d$, por consiguiente, basta igualar a la unidad la derivada de la función $\delta$ respecto de $d$, para hallar la deformación total en la situación de espesor límite.

Derivando $\delta$ respecto de $d$ e igualando a 1, se tiene:

$$
\delta^{\prime}=\frac{3 \cdot 27 \cdot w_{d} \cdot h^{4}}{128 \cdot\left(t_{d}-d\right)^{4} \cdot E}=1
$$

Haciendo $\delta=d$ en [8] se tiene:

$$
d=\frac{27 \cdot w_{d} \cdot h^{4}}{128 \cdot\left(t_{d}-d\right)^{3} \cdot E}
$$

Sustituyendo en [9]:

$$
\delta^{\prime}=\frac{3 \cdot d}{\left(t_{d}-d\right)}=1 ; d=\frac{t_{d}}{4}
$$

En la situación límite la deformación total es la cuarta parte del espesor de cálculo. Esta conclusión está recogida en el Documento Básico Seguridad Estructural: Fábrica (en adelante, $D B S E-F)$.

Sustituyendo en [9] el valor obtenido de la deformación ( $d=$ $\left.t_{d} / 4\right)$ se puede obtener, además, el valor de la esbeltez en la situación límite (en adelante, esbeltez crítica) (3):

\footnotetext{
9 Este fenómeno no es otro que el conocido como «pandeo», en el que la semilla de excentricidad es, en este caso, la flecha por acción horizontal.
} 


$$
\begin{gathered}
3 \cdot 27 \cdot w_{d} \cdot h^{4}=128 \cdot\left(\frac{3}{4}\right)^{4} \cdot t_{d}^{4} \cdot E \\
\left(\frac{h}{t_{d}}\right)_{\text {crítica }}^{4}=\frac{0,5 \cdot E}{w_{d}}
\end{gathered}
$$

De nuevo aparece la esbeltez como el parámetro determinante en el análisis de una fachada confinada. En este caso la esbeltez crítica opera elevada a la cuarta potencia, lo que indica que su influencia es decisiva en el comportamiento mecánico.

Si la esbeltez del muro confinado supera el valor de la esbeltez crítica para la acción considerada, el problema diverge, lo que significa un fallo por inestabilidad, sin que la fábrica tenga oportunidad de exhibir su resistencia a compresión. Esto supone una fuerte restricción a la resistencia por efecto arco de las fachadas, cuando la entrega en el forjado se realiza parcialmente para poder chapar el frente de los mismos con plaquetas. Si, además, se pretende disponer una barrera de aislamiento en el canto del forjado para evitar el puente térmico, la limitación de la esbeltez de la fachada para los valores habituales de la succión de viento convierte la solución en inviable con muros de ladrillo de $1 / 2$ pie de espesor.

\subsection{Soluciones alternativas a la fachada confinada}

El recurso de confinar las fachadas de ladrillo entre los forjados se impuso sin un marco normativo de respaldo, que recogiera el cambio sustancial en el comportamiento de estos elementos ${ }^{10}$.

La respuesta mecánica de los muros de fachada frente a la acción de viento por efecto arco es espontánea, pero requiere un buen confinamiento en los forjados asegurando el retacado de la última hilada del muro, y que los forjados de piso tengan la carga necesaria para contrarrestar el empuje. El análisis de la deformación indica que se precisa, además, un ancho de entrega mínimo del muro en los forjados, determinado en función de la altura del paño que, para fachadas de $1 / 2$ pie, sólo está disponible en situaciones de altura de planta modestas o succión de viento poco agresiva. Pero, además, el hecho de confinar la fachada entre los forjados exige la consideración de esta circunstancia en el análisis global de la estructura del edificio, puesto que ambos elementos, estructura y muro, quedan rígidamente conectados.

La falta de un análisis estructural adecuado para este tipo constructivo hizo que, en muchos casos, alguna de las condiciones anteriores resultara escasa o inexistente, lo que tuvo como consecuencia la aparición de disfunciones y procesos patológicos en las fachadas que nunca se habían producido en los muros de carga.

Los procesos patológicos son de índole diferente, según el requisito que resulta deficitario en cada situación particular, pero en la mayoría de los casos se solían atribuir a la acumulación de carga sobre los muros de fachada o a su espesor reducido, cuando en realidad ninguno de los procesos patológicos habituales en las fachadas de ladrillo (fisuración en esquinas y dinteles, caídas de petos, desprendimiento de plaquetas, etc.) constituyen un síntoma de agotamiento por sobrepeso, sino todo lo contrario. Más aún si se tiene en cuenta que estos procesos aparecen prioritariamente en las plantas altas de los edificios.

\subsubsection{Solución con perfiles angulares intercalados}

En las situaciones en las que no se disponía de una entrega suficiente del muro en los forjados, o de forma sistemática, intentando evitar las incertidumbres inherentes al proceso constructivo de las fachadas confinadas, se impuso el recurso de intercalar perfiles angulares, concebidos erróneamente como «elementos de apoyo», en los frentes de forjado. Estos perfiles de acero quedaban parcialmente embutidos en el muro a nivel de cada planta.

El resultado de este procedimiento no siempre produjo los efectos deseados, porque el dimensionado de los elementos auxiliares, no procede de ningún análisis estructural ${ }^{11}$; además de suponer un innecesario encarecimiento de la solución constructiva, y la aparición de otros efectos secundarios que las fachadas confinadas no tenían, como por ejemplo el abombamiento y desprendimiento de piezas por oxidación del perfil intercalado.

La disposición de angulares pretendía restituir una falta de «apoyo» en los forjados, cuando en realidad la condición de apoyo (entendiendo como tal la respuesta frente a la carga gravitatoria) sólo se precisa en la planta de arranque.

Por otra parte, el empeño en trasmitir el peso de la fachada a los forjados, planta a planta, no tiene ninguna ventaja estructural para el muro de ladrillo; aparte de ser un empeño vano porque cuando existe continuidad en vertical del muro éste gravita sobre sí mismo en cualquier caso, debido a la enorme diferencia de rigidez, ante carga vertical, entre muro y forjado. Aunque el muro de fachada esté interrumpido de trecho en trecho por los forjados, la carga pasa de manera transparente a través de ellos, siempre que haya algo de continuidad. Esta circunstancia está explícita en el Documento Básico Seguridad Estructural: Acciones en la Edificación ${ }^{12}$.

El parámetro deficitario en las fachadas confinadas no es la falta de «apoyo» sino la falta de «entrega» contra los forjados cargados, cuyo valor mínimo se determina según [12], en función de la altura y la succión de viento ${ }^{13}$.

El hecho de intercalar un angular, si éste no está implicado en la carga gravitatoria del forjado, no tiene ningún efecto en el análisis según el modelo arco. Si se utiliza el modelo viga

${ }^{10}$ Hasta la entrada en vigor del CTE la única Norma Básica Española que recogía los muros de ladrillo era la Norma Básica de la Edificación FL-90, que se refería exclusivamente a los muros de carga, cuyas rutinas y modelos no sirven para aplicarlos a los muros de fachada.

${ }^{11} \mathrm{El}$ angular se dimensionaba por razones de compatibilidad geométrica sin el menor cálculo estructural de respaldo. La regla al uso consistía en disponer un perfil cuya longitud de ala permitiera restituir un ancho de entrega del ladrillo no inferior a 2/3 de su espesor y, simultáneamente, que el ancho del ala no sobrepasara el espesor de las plaquetas.

${ }^{12}$ Hasta la aprobación del CTE era muy habitual que se interpretara, sin demasiado fundamento, que los muros de fábrica son carga para la estructura de la planta donde están dispuestas. Aún hoy día subsiste esta creencia como superstición.

${ }^{13}$ Las reglas, ya en desuso, que determinaban el ancho de entrega como una fracción del grueso del muro (por ejemplo, mínimo 2/3) carecen de fundamento. La entrega mínima necesaria es una función de la altura del paño, y no del grueso del muro. 
vertical para equilibrar la acción horizontal, se puede prescindir del empuje vertical en la reacción, pero entonces el muro fracasa por flexión vertical para valores muy inferiores a los procedentes de acción habitual del viento. Los tacos de fijación del angular a los forjados, si se dimensionan adecuadamente, pueden resolver la estabilidad del muro frente al vuelco, pero no resuelven su resistencia.

Cuando el ancho de entrega del muro en los forjados es deficitario, sólo se puede justificar la validez de la solución estructural utilizando para el análisis el modelo placa, con flexión en dos direcciones. Pero este modelo precisa que el muro esté eficazmente anclado a los soportes, y el recurso del angular intercalado se impuso sin tener en cuenta esta circunstancia.

\subsubsection{Solución con juntas horizontales de movimiento}

Con el objetivo de evitar los problemas de incompatibilidad de deformaciones entre las fachadas de ladrillo y la estructura del edificio en la que se sustentan, o la incertidumbre acerca del posible trasvase de carga gravitatoria por diferencia de rigidez, surgió, como solución alternativa al confinamiento, el recurso de interrumpir la continuidad vertical del muro disponiendo juntas horizontales de movimiento, cada una o dos plantas. Con esta solución habitualmente el muro no se sustenta directamente sobre los forjados, sino que se separa de la estructura descargando en dispositivos específicos de apoyo, tales como consolas o ménsulas interpuestas entre el forjado y la hoja exterior del cerramiento.

Estos sistemas de sustentación resuelven la incompatibilidad de movimientos entre la fachada y la estructura, y habilitan, aunque sólo parcialmente, la continuidad del resto de los componentes del cerramiento, para evitar los engorrosos puentes. Sin embargo, la presencia de juntas horizontales de movimiento elimina la posibilidad de generar empujes contra los forjados y, consecuentemente, elimina también la posibilidad de resistir acciones horizontales por efecto $\operatorname{arco}^{14}$. El equilibrio ante la acción de viento sólo se puede conseguir mediante un análisis según el modelo placa, pero en unas condiciones de resistencia y estabilidad muy mermadas respecto a las que tiene un muro con continuidad en vertical.

La penalización que supone para el análisis la interrupción de la continuidad en vertical del muro mediante juntas horizontales es doble. Por una parte, la condición de sustentación del muro queda sustancialmente disminuida al ser posible el giro en la cabeza ${ }^{15} \mathrm{y}$, por otra parte, la capacidad resistente a flexión vertical de la fábrica (con plano de rotura paralelo a los tendeles) se reduce notablemente al eliminar el efecto beneficioso que supone el peso propio del muro de las plantas superiores.

Por ello, para que el análisis de esta solución resulte satisfactorio, se necesita recurrir a la resistencia por flexión horizontal de la fábrica (con plano de rotura perpendicular a los tendeles), lo cual sólo es posible si el muro está eficazmente anclado a los pilares. Si éstos no están muy próximos, como es lo habitual en las estructuras de pórticos de hormigón o acero, se precisa, además, una importante cuantía de armadura de tendel para suministrar la resistencia a tracción horizontal, de la que el muro es deficitario.

Los sistemas que utilizan juntas horizontales de movimiento son viables y eliminan las incertidumbres inherentes al sistema convencional relacionadas con el trasvase de carga entre forjados y muro, pero a costa de consumir una gran cantidad de recursos auxiliares y de requerir un análisis de la estabilidad muy afinado por tener sustancialmente reducidas las condiciones de sustentación.

\section{RAZÓN DE SER DE LA FACHADA AUTOPORTANTE}

Según se ha expuesto en todos los casos precedentes el comportamiento mecánico de un muro de fachada está determinado por su escasa resistencia a tracción. Por el contrario, la resistencia a compresión siempre sobra en el análisis. La tensión de compresión debida al propio peso de un muro de fachada de ladrillo, con un peso específico de $18 \mathrm{kN} / \mathrm{m}^{3}$, y con alturas de piso del orden de 3,00 $\mathrm{m}$, tiene un valor de cálculo de $18 \mathrm{kN} / \mathrm{m}^{3} \cdot 1,35 \cdot 3,00 \mathrm{~m}=0,073 \mathrm{~N} / \mathrm{mm}^{2}$ por planta, lo que representa menos del $5 \%$ de la tensión soportable por el material.

Por otra parte, siempre que el rango de la tensión de compresión se mantenga por debajo de la mitad de la resistencia del material ${ }^{16}$, el efecto de la acción gravitatoria es beneficioso. En las fachadas confinadas la presencia de forjados cargados es imprescindible para generar empujes, y en las fachadas ancladas la acción del peso propio es beneficiosa para contrarrestar tracciones. El recurso de anclar un muro puede resolver su estabilidad, pero no resuelve su resistencia. La compresión resuelve tanto la estabilidad como la resistencia.

La manera más sencilla y natural de proveer parte de la carga estabilizante que precisa un muro de fachada ante las acciones horizontales, si hay plantas por encima, es utilizar su propio peso. Y esto se consigue de forma espontánea, sin incremento del coste ni de la complejidad constructiva de la solución.

Otra conclusión que se puede extraer del análisis es que la continuidad vertical del muro, tanto geométrica como mecánica, también tiene un efecto favorable en su respuesta estructural. La continuidad geométrica es favorable porque permite contabilizar el propio peso del muro en toda su altura, y la continuidad mecánica es favorable porque reduce sustancialmente los esfuerzos de flexión.

Estas importantes conclusiones y una mirada hacia atrás, observando la forma de concebir los muros de ladrillo que tenían nuestros mayores, son suficientes motivos para reconsiderar el procedimiento de resolver las fachadas de ladrillo de los edificios modernos.

\footnotetext{
${ }_{14}$ Esta advertencia está explícita en el DB SE-F, artículo 5.2 «Muros con acciones laterales locales», párrafo 2.

${ }_{15}$ La continuidad en los elementos solicitados a flexión equivale una reducción de la luz de flexión del 30 \%, y a una reducción de los esfuerzos máximos del $50 \%$, respecto de los elementos con la continuidad interrumpida.

${ }^{16}$ Esta circunstancia se produce en fachadas de edificios de menos de 10 plantas.
} 


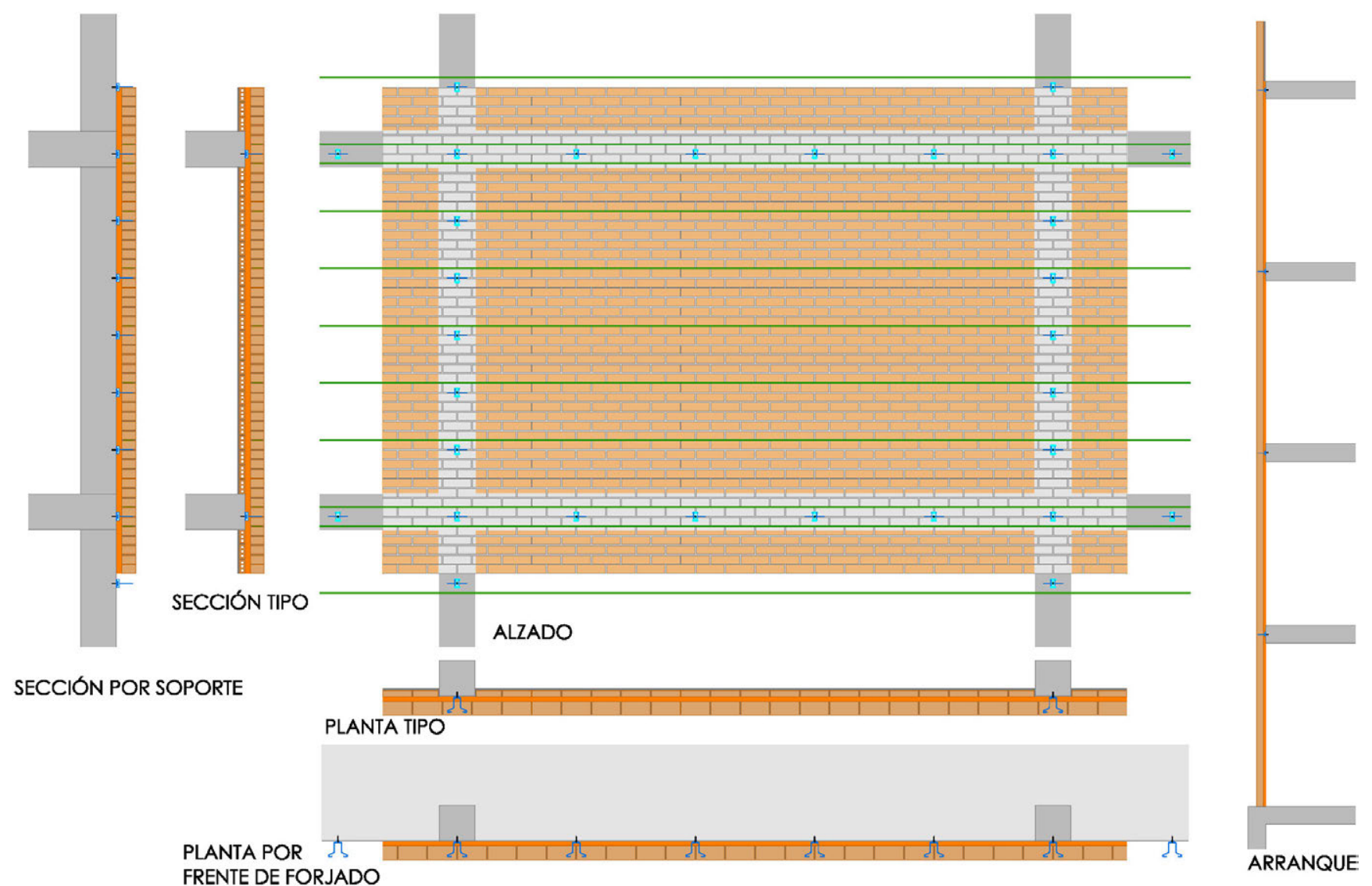

Figura 4. Descripción constructiva de la 'Fachada Autoportante' de ladrillo cata vista.

Por una parte, cabe pensar que, si la fachada se concibe como una piel del edificio, es una contradicción encastrarla en su estructura, porque tarde o temprano acabará participando de su comportamiento mecánico. Por otra parte, si la piel está constituida por un material pétreo con suficiente porte y capacidad para resistir incluso carga ajena, no hay ninguna razón para rehuir su propio peso intentando disiparlo en cada planta del edificio con difíciles detalles de encuentro o costosos artilugios de apoyo.

Éstas son las razones que dieron origen al tipo constructivo que aquí se ha llamado 'Fachada Autoportante', cuya principal ventaja es la incorporación del potencial del material cerámico en el análisis estructural.

\subsection{Descripción del tipo constructivo}

La solución constructiva de la 'Fachada Autoportante' consiste en separar la hoja exterior del cerramiento de la estructura del edificio, permitiendo el paso de una cámara continua con aislamiento (Figura 4).

Con esta disposición pasante del muro de fachada se consiguen dos ventajas fundamentales: mejorar el comportamiento higrotérmico del cerramiento, y eliminar el conflicto constructivo que supone el confinamiento en los forjados.
El tipo de 'Fachada Autoportante' se incluye dentro de las soluciones avanzadas o novedosas, aunque esta consideración en rigor sólo es cierta desde el punto de vista del proceso constructivo y de las implicaciones que tiene en la eficiencia energética del edificio. Desde el punto de vista estructural, el comportamiento mecánico de la 'Fachada Autoportante' no difiere esencialmente del comportamiento de otras soluciones pretendidamente convencionales ${ }^{17}$.

El aislamiento térmico puede alojarse en la cámara continua, o puede colocarse adosado a la hoja interior del cerramiento, revistiendo previamente con material aislante los frentes de soportes y forjados; en este último caso no se precisa la continuidad de la cámara, y la hoja exterior del cerramiento puede colocarse tangente a la estructura del edificio, sin separación significativa entre ambos elementos.

A su vez, la cámara de aire puede estar ventilada si las condiciones higrotérmicas lo aconsejan. La ventilación de la cámara se consigue simplemente dejando llagas sin rellenar en la primera y última hiladas. Esta situación corresponde a la solución de «fachada ventilada» con acabado de ladrillo tradicional (4). La característica esencial de la 'Fachada Autoportante' es su continuidad al paso por los forjados, gravitando sobre sí misma en toda la altura que permite el análisis estructural. La sustentación en el arranque se realiza sobre un elemento estructural que, a efectos cálculo, se puede considerar de

\footnotetext{
${ }^{17}$ La solución de fachada confinada, incluso disponiendo angulares auxiliares en cada planta, exige considerar que el muro gravita sobre sí mismo, en virtud de la continuidad en vertical, según el Documento Básico «Acciones en la edificación», artículo 2.1. «Peso propio», párrafo 5; por lo que el muro debe resistir en la planta de arranque el efecto de su propio peso correspondiente a toda la altura de la fachada.
} 
rigidez infinita. Esta situación puede corresponder al arranque de la fachada sobre la cabeza de un muro de sótano, una solera de hormigón o una zapata corrida de cimentación. Si hubiere soportales o grandes huecos en planta baja, el muro puede arrancar de una viga cargadero, con suficientes puntos de anclaje a la estructura del edificio para poder simular en el análisis un apoyo infinitamente rígido ${ }^{18}$.

La estabilidad frente a la tendencia al vuelco del muro se resuelve con anclajes de retención que se fijan en los frentes de forjados y soportes, y que deben tener suficiente resistencia para suministrar la totalidad de la reacción horizontal correspondiente a la acción de viento, según el DB SE-F, artículo 5.5 «Llaves». Tienen, además, la misión de reducir la esbeltez de los paños frente al fenómeno del pandeo. El diseño de los dispositivos de anclaje debe permitir libertad de movimiento vertical, para evitar así el trasvase de carga gravitatoria entre los forjados o soportes y el muro. De esta forma, la carga gravitatoria del muro de fachada está perfectamente determinada puesto que sólo es la procedente de su propio peso, por lo que su efecto puede ser introducido en el análisis sin ningún tipo de incertidumbre, tanto si resulta favorable para el muro, como si resulta desfavorable para el elemento estructural del arranque.

\subsection{Modelo para el análisis de las 'Fachadas Autoportantes'. Modelo placa}

El tipo estructural de referencia para verificar el comportamiento mecánico de una 'Fachada Autoportante' anclada a forjados y soportes es el correspondiente a una placa sustentada en bordes, con cargas perpendiculares a su plano.

El procedimiento de comprobación sancionado por el DB SE-F, artículo 5.4.2 "Análisis de solicitaciones en flexión», párrafo 6, es el método de las líneas de rotura, que se fundamenta en plantear la condición de equilibrio energético para la configuración de rotura pésima, de todas las posibles que sean compatibles con las condiciones de sustentación de los bordes. La condición de resistencia suficiente se obtiene realizando el balance entre el trabajo de la acción exterior en un recorrido arbitrario según el patrón de rotura analizado, y el trabajo interno que demanda la estructura para romper según las líneas definidas por ese mismo patrón.

El método de las líneas de rotura es un recurso muy potente para analizar elementos redundantes, aunque tiene el inconveniente de que hay que realizar tanteos para detectar la configuración de rotura pésima. Afortunadamente, para casos de geometría sencilla, como es el correspondiente a un paño rectangular, el patrón de rotura más simple (por ejemplo, rotura en cruz o en sobre) produce resultados muy próximos al pésimo, lo que permite formular (5) o tabular los resultados para diferentes condiciones de sustentación en los bordes (1).

Si se supone un paño de fachada, sustentando en los cuatro bordes, con continuidad, la condición de resistencia, por el método de las líneas de rotura se puede expresar de la siguiente forma (Figura 5):
Condición de resistencia: $W_{\text {ext }} \leq W_{\text {int }}$

con:

$$
\begin{gathered}
W_{\text {ext }}=w_{d} \cdot H \cdot L \cdot \frac{\delta}{3} \\
W_{\text {int }}=2 \cdot\left[\left(m_{1}^{-}+m_{1}^{+}\right) \cdot L \cdot \frac{\delta}{\frac{H}{2}}+\left(m_{2}^{-}+m_{2}^{+}\right) \cdot H \cdot \frac{\delta}{\frac{L}{2}}\right]
\end{gathered}
$$

Sustituyendo en [13]:

$$
w_{d} \cdot H \cdot L \leq 12 \cdot\left(m_{1} \cdot \frac{L}{H}+m_{2} \cdot \frac{H}{L}\right)
$$

siendo:

$w_{d} \quad$ valor de cálculo de la acción horizontal;

$H$ altura del paño entre filas de anclajes;

L longitud del paño entre columnas de anclajes;

$m_{1} \quad$ capacidad resistente del muro a flexión vertical por unidad de longitud $\left(=m_{1}^{-}=m_{1}^{+}\right)$;

$m_{2}$ capacidad resistente del muro a flexión horizontal por unidad de altura $\left(=m_{2}^{-}=m_{2}^{+}\right)$

Las capacidades resistentes del muro $\left(m_{1}, m_{2}\right)$ a flexión vertical y horizontal, respectivamente, se obtienen, en primera aproximación, como el producto del módulo resistente por las respectivas resistencias a flexión, cuyos valores, por defecto, se pueden tomar del orden de $160 \mathrm{kN} / \mathrm{m}^{2}$, en valor de cálculo, lo que arroja resultados bajos de capacidad resistente cuando se trata de un muto de ladrillo de $1 / 2$ pie de espesor.

La posibilidad de usar este modelo de análisis con los valores habituales de acción de viento y dimensiones razonables de los paños de fachada, está condicionada al empleo de recursos adicionales para conseguir suficiente resistencia a flexión en el muro.

La resistencia a flexión horizontal (con plano de rotura perpendicular a los tendeles) se puede incrementar incorporando armadura de tendel. Con este recurso, utilizado con las cuantías mínimas que establece el DB SE-F, artículo 7.5 «Disposiciones relativas a las armaduras», se consigue, además, dotar de cierta ductilidad al muro, lo que permite aplicar los modelos de respuesta que suponen plastificación de secciones.

La resistencia a flexión vertical (con plano de rotura paralelo a los tendeles) se puede aumentar sustancialmente si existe tensión normal de compresión, debida al peso propio, que se suma al valor de la resistencia, incrementando en la misma medida su valor.

Lógicamente, el recurso de incorporar peso al muro de fachada tiene un límite, determinado por la resistencia a compresión

\footnotetext{
${ }^{18}$ En el caso de arranque sobre elementos a flexión (viga de forjado o viga cargadero) la condición de rigidez infinita supone limitar la flecha del elemento al valor de 1/1.00o de la luz.
} 


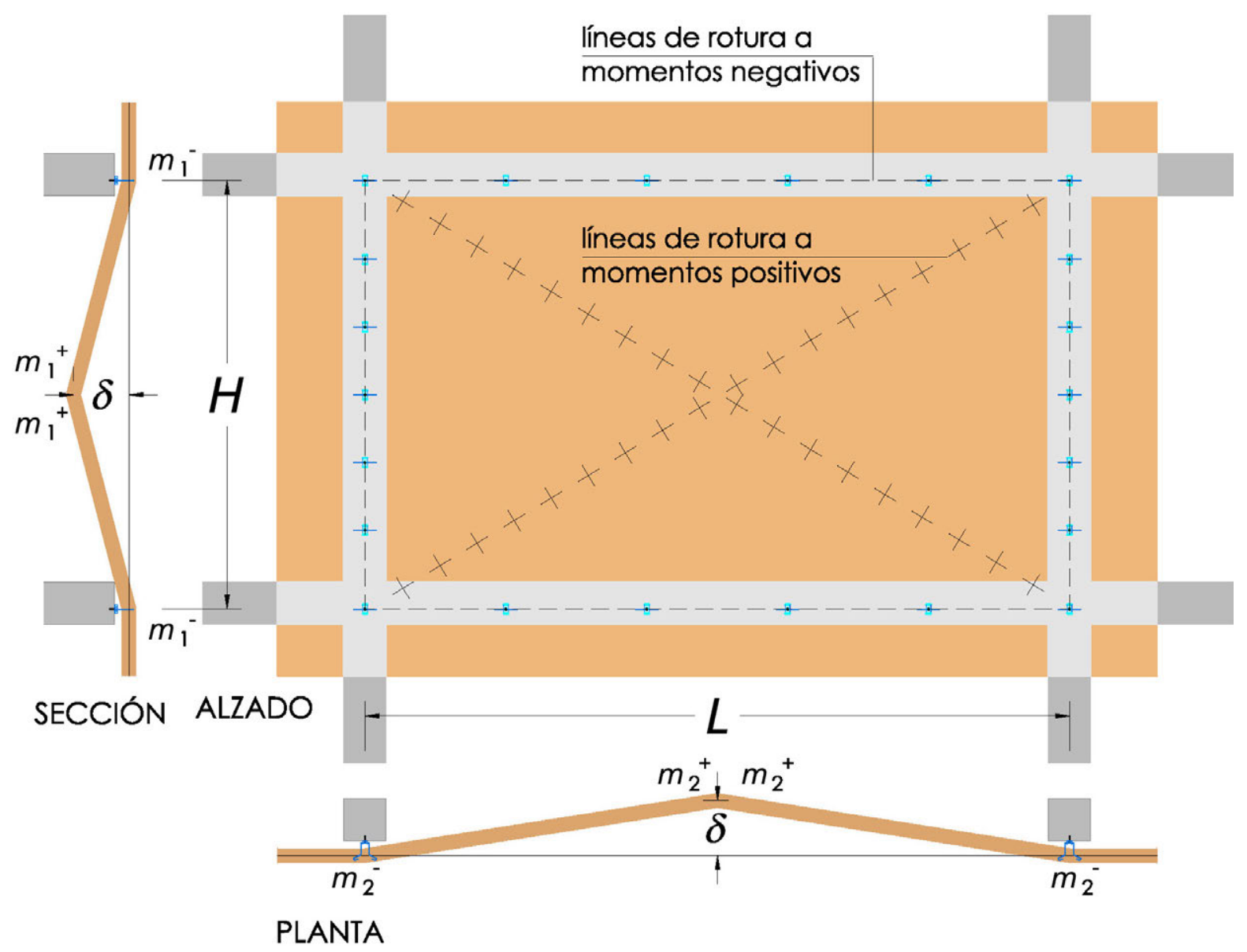

Figura 5. Esquema para el análisis de un paño de fachada según el modelo placa. Configuración de rotura.

de la fábrica con la correspondiente penalización que supone el pandeo. También en este caso, es la esbeltez el parámetro geométrico que determina la solución del problema, cuyo control se consigue disponiendo suficientes anclajes de retención.

\section{CONCLUSIONES}

Los diferentes modelos de análisis expuestos anteriormente permiten conocer la influencia que tienen las distintas variables (geométricas y mecánicas) en las prestaciones estructurales de los muros de fachada, destacando en todos ellos el efecto beneficioso de dos parámetros fundamentales: las condiciones de sustentación en continuidad y la presencia de carga gravitatoria.

La principal conclusión para utilizar en la fase de proyecto de una fachada de ladrillo cerámico es que las verdaderas variables que determinan el comportamiento mecánico son de índole geométrica, mientras que la resistencia del material tiene poca trascendencia en los resultados. La resistencia a compresión es tan elevada con relación a la solicitación que, incluso con la penalización que supone el pandeo, sólo tiene trascendencia para el dimensionado en fachadas de un número superior a diez plantas, y sólo si se trata de paños con luces superiores a 6,00 m (1). La resistencia a flexión vertical es prácticamente un invariante, con un valor tan bajo que sólo tiene carácter testimonial, y la resistencia a flexión horizontal, aunque es algo superior, sólo se contabiliza en fábricas no armadas.

El parámetro geométrico que condiciona el dimensionado, en todos los casos, es la esbeltez del muro, siendo decisiva en las soluciones convencionales, y de especial relevancia en la solución autoportante. La condición de esbeltez, como limitación general de índole geométrica para los muros de fábrica, está presente en todas las normas consultadas sobre la materia. En el ámbito nacional, el DB SE-F impone límites de esbeltez a los muros sólo cuando son de carga o cuando están solicitados a flexión procedente de carga vertical (muros trabajando como vigas o dinteles). Sin embargo, la normativa de ámbito europeo (6) e internacional (7) establece límites explícitos de esbeltez para los muros con función exclusiva de cerramiento.

Los factores determinantes para el dimensionado de los muros de fachada, a saber, esbeltez y presencia de carga gravitatoria, también son determinantes para los muros de carga tradicionales, cuya función es el sostén del edificio. La continuidad en vertical, imprescindible para los muros de carga por razones obvias, se manifiesta también como una condición esencial para la resistencia y estabilidad de los muros cuya función es sólo de cerramiento.

El tipo constructivo de 'Fachada Autoportante' de ladrillo constituye una muestra de las numerosas posibilidades que ofrecen los materiales cerámicos tradicionales para ser utilizados en los elementos constructivos de los edificios modernos, incorporando en el análisis las cualidades propias del material. Constituye también un ejemplo de que el análisis sistemático de diferentes soluciones alternativas, es un procedimiento que permite incrementar las prestaciones de los elementos constructivos sin incrementar necesariamente el coste del producto final. 


\section{REFERENCIAS}

(1) Vega, L., y otros. (2008). Catálogo de soluciones cerámicas para el cumplimiento del Código Técnico de la Edificación. Anejo A.5, pp. 359-367. Hispalyt.

(2) Unión Europea (2010). Directiva 2010/31/UE del Parlamento Europeo y del Consejo de 19 de mayo de 2010 relativa a la Eficiencia energética de los edificios. Diario Oficial de la Unión Europea, L 153/13-35.

(3) Sastre, V. (2006). Comportamiento de un muro confinado ante acciones laterales. Revista Conarquitectura (20): 81-88.

(4) Paricio, I. (1995). La fachada ventilada con ladrillo cara vista. Revista Nueva Arquitectura con Arcilla Cocida (2): 37.

(5) Estévez, J., Otero, D. (2006). Cálculo de muros de fábrica sometidos a cargas laterales de viento. Hormigón y Acero (240): 55-62.

(6) AENOR. Eurocódigo 6: Proyecto de estructuras de fábrica. Parte 3. UNE-EN 1996-3. Anejo C.

(7) Building Code Requirements for Masonry Structures. ACI 53O-O5/ASCE 5-O5/TMS 402-05. Chapter 5. "Empirical design of masonry", pp. 45-49. 